\title{
Effect of nodule shape for modeling of auxetic microporous polymers
}

\author{
Teik-Cheng Lim \\ SIM University, Singapore
}

\begin{abstract}
Previous models for describing auxetic microporous polymers adopt 2D rectangular blocks with fibril interconnections. In this paper, spherical nodes are used in order to approximate the granular nodule shape and for taking into consideration the 3D nature of the nodes. Assuming curvilinear motion of the nodes as a result of fibril rotation, a quantitative description of the Poisson's ratio is given as a function of the nodule density packing factor. Comparison with the 3D rectangular model shows that the spherical model gives a more conservative description of the Poisson's ratio although both models exhibit similar trend. Results reveal the significance of the assumed node shape for modeling the Poisson's ratio of auxetic microporous polymers.
\end{abstract}

\section{Introduction}

Auxetic materials possess negative Poisson's ratios. Ever since the pioneering works on auxetic materials by Lakes et al $[1,2]$, Evans et al $[3,4]$ and Wojciechowski et al $[5,6]$, the field of auxetic materials has gained considerable prominence. The various geometrical models, experimental verifications and proposed applications have been summarized by Lim [7-41]. Due to the granular shape of the nodules, this paper develops a set of relationship between the Poisson's ratio and the density packing factor using spherical model in hexagonal array. For comparison with the rectangular geometry, a 3D rectangular model was also developed under the same set of criteria imposed upon the spherical model.

\section{Analysis}

Arising from the granular shapes of the nodules, they are herein modeled as spheres. Assuming the size variation of the nodules to be insignificant, hexagonal array of spheres is proposed. As such, a cross-sectional view for one plane that intersects the center of the sphere models is shown in Fig.1(a). Recall that in the rectangular model, the fibrils connect the edges of the rectangles [21-24] as shown in Fig.1(b). An equivalence for such connection in the case of spherical model is where the fibrils are tangential to the spheres as depicted in Fig.1(a). As a result of the hexagonal arrangement of the spheres, the angle between three mutually neighboring spheres is $60^{\circ}$, as shown in Fig.2. This schematic also shows the sphere radius $R$, the fibril length $l$ that connects $\mathrm{A}$ and $\mathrm{B}$, and the angle $\theta$ between the fibril and the horizontal axis. It is further assumed that the fibrils act as rigid rods, which permit curvilinear motion of the nodules.

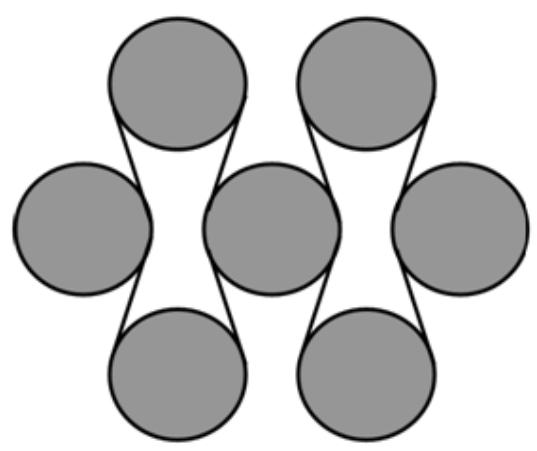

(a)

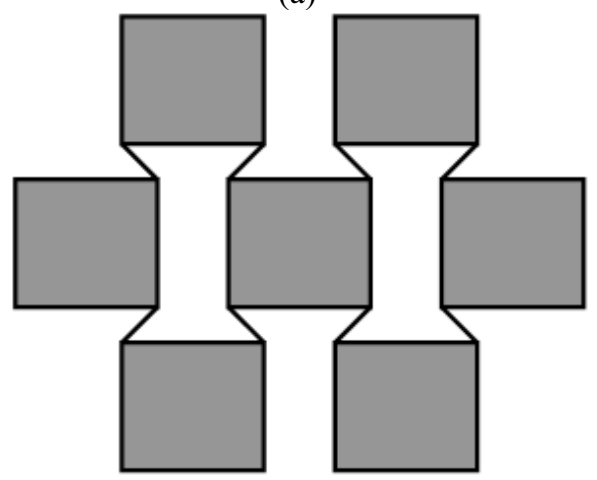

(b)

Figure 1. Hexagonal array of (a) spherical nodules and (b) rectangular nodules.

To develop a relationship between the Poisson's ratio and the density packing factor, it is assumed that the density packing factor of a hexagonal closed pack (HCP) to be similar to that of the face-centered cubic (FCC) whereby the arrangements are $\mathrm{ABABAB}$ and $\mathrm{ABCABC}$ respectively. With reference to Fig.2,

$$
(\overline{O C})=\sqrt{4 R^{2}+l^{2}}
$$

Corresponding author: tclim@unisim.edu.sg 


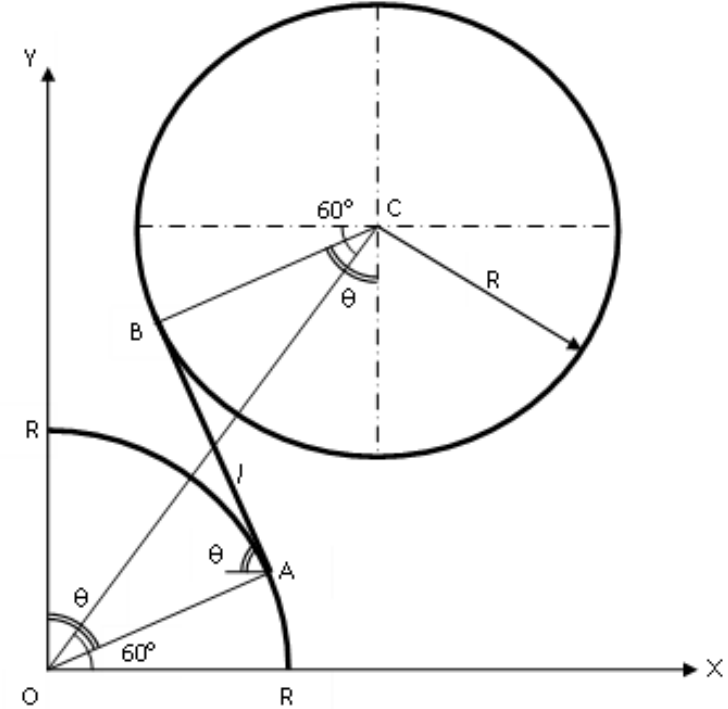

Figure 2. Schematic representation of spherical nodule for analysis

The density packing factor can be taken as the ratio of the spherical nodule within the boundary of an FCC representative volume element (RVE)

$$
V_{\text {sphere }}=\frac{16}{3} \pi R^{3}
$$

to the volume of the RVE itself

$$
V_{R V E}=2 \sqrt{2}(\overline{O C})^{3} \text {. }
$$

Therefore the density packing factor of hexagonally arranged spheres is

$$
d_{s p h}=\frac{8 \pi}{3 \sqrt{2}}\left(4+(l / R)^{2}\right)^{-\frac{3}{2}}
$$

A clockwise rotation of fibril $\mathrm{AB}$ by an infinitesimal angle $d \theta$ gives a relative displacement of $\mathrm{B}$ with respect to $\mathrm{A}$ as

$$
x_{B / A}=l \sin \theta d \theta=x_{C / O}
$$

and

$$
y_{B / A}=l \cos \theta d \theta=y_{C / O}
$$

resolved along the $\mathrm{x}$ - and $\mathrm{y}$-axes respectively. Assuming the spheres to be rigid, the displacement of $\mathrm{C}$ with respect to $\mathrm{O}$ is equal to that of $\mathrm{B}$ with respect to $\mathrm{A}$. This translates to the strains

$$
\varepsilon_{x}=\frac{x_{C / O}}{\overline{O C} \cos (\pi / 3)}=\frac{2 l \sin \theta d \theta}{\sqrt{4 R^{2}+l^{2}}}
$$

and

$$
\varepsilon_{y}=\frac{y_{C / O}}{\overline{O C} \sin (\pi / 3)}=\frac{2 l \cos \theta d \theta}{\sqrt{3} \sqrt{4 R^{2}+l^{2}}},
$$

and hence the Poisson's ratio according to the spherical model

$$
v_{s p h}=-\frac{\varepsilon_{y}}{\varepsilon_{x}}=-\frac{1}{\sqrt{3} \tan \theta} .
$$

Perusal to the geometry shown in Fig.2 shows that
$\overline{O C}\left\{\begin{array}{l}\cos (\pi / 3) \\ \sin (\pi / 3)\end{array}\right\}=\left[\begin{array}{cc}\sin \theta & -\cos \theta \\ \cos \theta & \sin \theta\end{array}\right]\left\{\begin{array}{c}2 R \\ l\end{array}\right\}$,

therefore

$$
\tan \theta=\frac{2 R+\sqrt{3} l}{2 \sqrt{3} R-l}
$$

which leads to

$$
v_{s p h}=\frac{(l / R)-2 \sqrt{3}}{3(l / R)+2 \sqrt{3}} .
$$

To express the Poisson's ratio in terms of density packing factor, Eq.(4) is rewritten as

$$
\frac{l}{R}=\sqrt{\left(\frac{4 \sqrt{2} \pi}{3 d}\right)^{\frac{2}{3}}-4}
$$

for substitution into Eq.(12). The Poisson's ratio for spherical model can be compared with that of the $3 \mathrm{D}$ rectangular block model in order to observe the discrepancy arising from the assumed nodule shape. For the comparison to be appropriate, there is a need for the rectangular blocks to be arranged in $\mathrm{HCP}$ as in the case of the spherical model. See Fig.3(a).

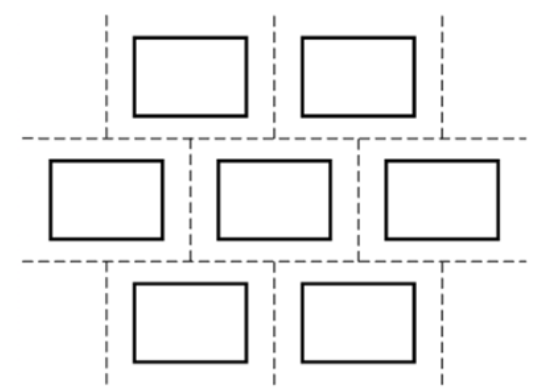

(a)

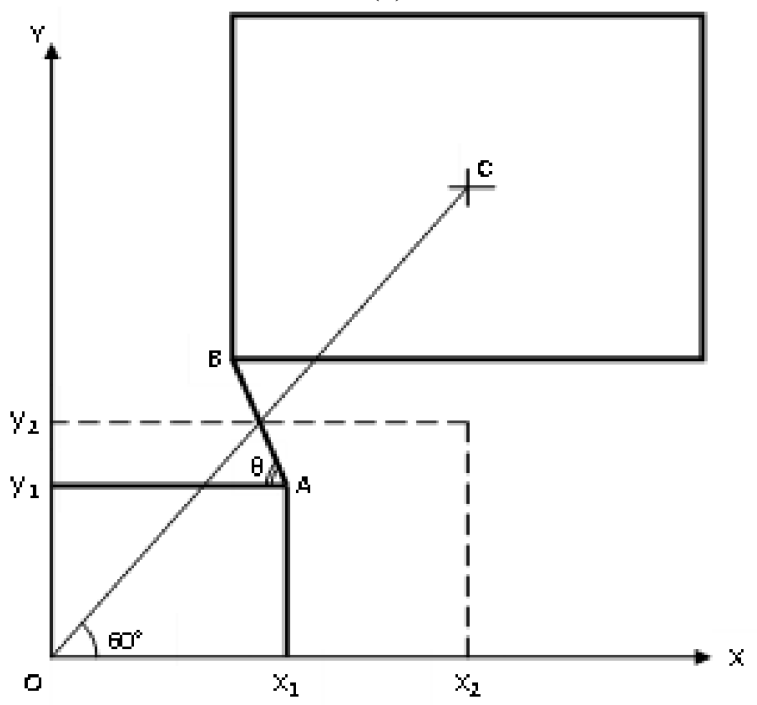

(b)

Figure 3. (a) Hexagonal array of rectangular nodules, denoted by bold rectangles, within their respective representative volume elements (RVE), denoted by dashed boundary, and (b) schematics for analysis of 3D rectangular blocks in hexagonal array. 
For the $3 \mathrm{D}$ rectangular block to be in proportion with its surrounding space, the length-to-width ratio is

$$
\frac{x_{1}}{y_{1}}=\frac{x_{2}}{y_{2}}=\frac{2}{\sqrt{3}} \text {. }
$$

If load is applied in the $\mathrm{x}$-direction, we assume $y_{1}=z_{1}$ and $y_{2}=z_{2}$ such that the density packing factor is

$$
d_{r e c}=\frac{x_{1}}{x_{2}}\left(\frac{y_{1}}{y_{2}}\right)^{2}=\left(\frac{y_{1}}{y_{2}}\right)^{3} \text {. }
$$

For the configuration shown in Fig.3(b), the displacement of $\mathrm{C}$ with respect to $\mathrm{O}$ is furnished in Eqs.(5) and (6). Hence the strains are

$$
\varepsilon_{x}=\frac{l \sin \theta d \theta}{x_{2}}=\tan \theta d \theta
$$

and

$$
\varepsilon_{y}=\frac{l \cos \theta d \theta}{2 y_{2}}=\left(1-\frac{y_{1}}{y_{2}}\right) \frac{d \theta}{\tan \theta}
$$

thereby leading to

$$
v_{r e c}=-\frac{\varepsilon_{y}}{\varepsilon_{x}}=\left(\frac{y_{1}}{y_{2}}-1\right) \frac{1}{\tan ^{2} \theta} .
$$

By the geometry described in Fig.3(b),

$$
\tan \theta=2 \frac{y_{2}-y_{1}}{x_{2}}
$$

therefore

$$
v_{\text {rec }}=-\frac{1}{3(1-\sqrt[3]{d})} .
$$

A comparison between the Poisson's ratios of 3D hexagonally arranged 3D rectangular and spherical nodules, and their auxeticity range are shown in Table 1.

Table 1. Expressions of Poisson's ratio as functions of density packing factor for hexagonal array of nodules

\begin{tabular}{|l|l|}
\hline $\begin{array}{l}\text { Rectangular } \\
\text { nodules }\end{array}$ & Spherical nodules \\
\hline$v_{\text {rec }}=\frac{1}{3(\sqrt[3]{d}-1)}$ & $v_{s p h}=\frac{p-2 \sqrt{3}}{3 p+2 \sqrt{3}}$ \\
\hline N.A. & $p=\sqrt{\left(\frac{4 \sqrt{2} \pi}{3 d}\right)^{\frac{2}{3}}-4}$ \\
\hline$\frac{1}{8}<d \leq 1$ & $\frac{1}{8}<\frac{3 \sqrt{2} d}{\pi} \leq 1$ \\
\hline
\end{tabular}

It is clear that the extreme conditions for the 3D rectangular model are

$$
v_{\text {rec }}=\left\{\begin{array}{ccc}
-\frac{1}{3} & ; & d=0 \\
-\infty & ; & d=1
\end{array} .\right.
$$

In the case of spherical model,

$$
v_{s p h}=\left\{\begin{array}{c}
+\frac{1}{3} ; d=0 \\
-1 ; d=\frac{\pi}{3 \sqrt{2}}
\end{array}\right.
$$

whereby $d=\pi /(3 \sqrt{2})$ is the highest density packing factor for rigid spheres.

\section{Results and Discussion}

It is obvious from Eqs.(21) and (22) that for affixed density packing factor, the spherical model predicts higher Poisson's ratio than that by the $3 \mathrm{D}$ rectangular model. To schematically demonstrate how the Poisson's ratio varies with the density packing factor under the generic arrangement of nodules shown in Fig.2 and 3(b), a pair of Poisson's ratio curves were plotted using Eqs.(12) and (13) for the spherical model, and Eq.(20) for the $3 \mathrm{D}$ rectangular model. As depicted in Fig.4, the discrepancy between both models increases with the density packing factor.

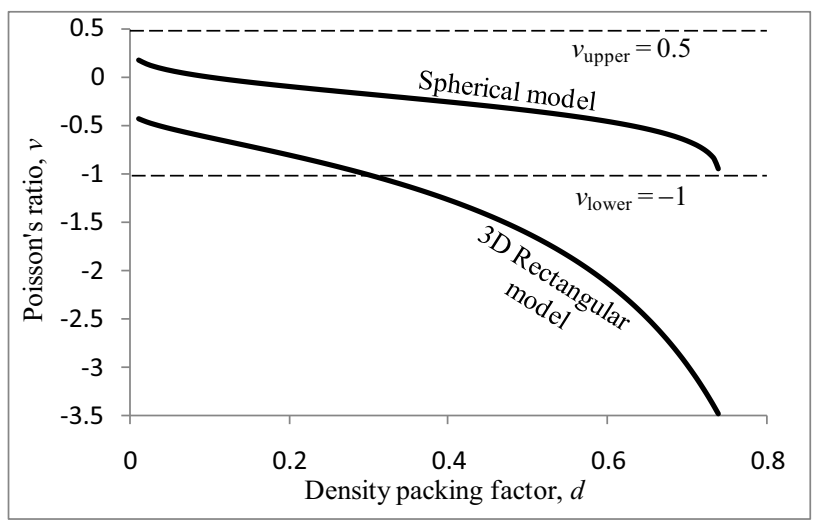

Figure 4. Comparison between the Poisson's ratio as a function of density packing factor for auxetic microporous polymers on the basis of spherical nodes and 3D rectangular nodes.

In spite of this discrepancy, it is can be observed that there is a qualitative agreement - a sharp drop in Poisson's ratios with increasing density packing factor for very small and very large packing factors. The drop in Poisson's ratios is more gradual for intermediate density packing factor. The observed discrepancy underlies the significance of the assumed nodule shape while the trend similarity verifies the validity of the spherical model with respect to the $3 \mathrm{D}$ rectangular model. Conversion of the $3 \mathrm{D}$ rectangular model to that of the spherical model in the case of 3D hexagonal array can be easily obtained from

$$
v_{s p h}=f_{c} v_{r e c}
$$

where the conversion factor is 


$$
f_{c}=(1-\sqrt[3]{d}) \frac{6-\sqrt{3} p}{2+\sqrt{3} p}
$$

with the parameter $p$ being defined as

$$
p=\sqrt{\left(\frac{4 \sqrt{2} \pi}{3 d}\right)^{\frac{2}{3}}-4} .
$$

\section{Conclusions}

A comparison between spherical and 3D rectangular models has been made for the Poisson's ratios of microporous polymers. With all other conditions fixed, the spherical model possesses a more conservative Poisson's ratio when compared to that of the $3 \mathrm{D}$ rectangular model. In spite of the discrepancy, a similar trend for the change in Poisson's ratios was observed with respect to the density packing factor. A conversion factor has been proposed to connect the Poisson's ratio based on the $3 \mathrm{D}$ rectangular model to that of the spherical model based on the $3 \mathrm{D}$ hexagonal arrangement of nodules.

\section{References}

1. R. Lakes, Science 235, 1038-1040 (1987)

2. R. Lakes, Science 238, 551 (1987)

3. B.D. Caddock and K.E. Evans, J. Phys. D: Appl. Phys. 22, 1877-1882 (1989)

4. K.E. Evans and B.D. Caddock, J. Phys. D: Appl. Phys. 22, 1883-1887 (1989)

5. K.W. Wojciechowski, Phys. Lett. A 137, 60-64 (1989)

6. K.W. Wojciechowski and A.C. Branka, Phys. Rev. A 40, 7222-7225 (1989)

7. T.C. Lim, J. Mater. Sci. 39, 4965-4969 (2004)

8. T.C. Lim, J. Mater. Sci. Lett. 22, 1783-1786 (2003)

9. T.C. Lim, J. Mater. Sci. 42, 7690-7695 (2007)

10. T.C. Lim, Phys. Status Solidi B 244, 910-918 (2007)

11. T.C. Lim, Eur. J. Mech. A/Solids 28, 752-756 (2009)

12. T.C. Lim and R. Acharya, J. Mater. Sci. 44, 44914494 (2009)
13. T.C. Lim, J. Eng. Mech. 136, 1176-1180 (2010)

14. T.C. Lim and R.U. Acharya, J. Biomed. Nanotechnol. 6, 718-724 (2010)

15. T.C. Lim and U.R. Acharya, J. Reinf. Plast. Compos. 29, 1441-1445 (2010)

16. T.C. Lim, J. Mater. Sci. 46, 6904-6909 (2011)

17. T.C. Lim, Phys. Status Solidi B 248, 140-147 (2011)

18. T.C. Lim and U. Rajendra Acharya, Phys. Status Solidi B 248, 60-65 (2011)

19. T.C. Lim, Phys. Status Solidi B 294, 1366-1372 (2012)

20. T.C. Lim, J. Mech. Med. Biol. 12, 1240027 (2012)

21. T.C. Lim, J. Mater.: Des. Appl. 227, 111-123 (2013)

22. T.C. Lim, Adv. Mater. Res. 804, 146-150 (2013)

23. T.C. Lim, Adv. Mater. Res. 804, 347-352 (2013)

24. T.C. Lim, J. Thermal Stresses 36, 1131-1140 (2013)

25. T.C. Lim, Smart Mater. Struct. 22084002 (2013)

26. T.C. Lim, Smart Mater. Struct. 22, 084001 (2013)

27. T.C. Lim, J. Mech. 29, 121-133 (2013)

28. T.C. Lim, Appl. Mech. Mater. 394, 134-139 (2013)

29. T.C. Lim, Mech. Res. Commun. 61, 60-66 (2014)

30. T.C. Lim, Adv. Mater. Res. 974, 398-401 (2014)

31. T.C. Lim, J. Eng. Mater. Technol. 136, 031007 (2014)

32. T.C. Lim, Int. J. Appl. Mech. 6, 1450012 (2014)

33. T.C. Lim, J. Eng. Mater. Technol. 136, 021007 (2014)

34. T.C. Lim, Smart Mater. Struct. 23, 045004 (2014)

35. T.C. Lim, A. Alderson, and K.L Alderson, Phys. Status Solidi B 251, 307-313 (2014)

36. T.C. Lim, P. Cheang and F. Scarpa, Phys. Status Solidi B 251, 388-396 (2014)

37. T.C. Lim, J. Eng. Mater. Technol. 137, 024502 (2015)

38. T.C. Lim, Mech. Adv. Mater. Struct. 22, 205-212 (2015)

39. T.C. Lim, Auxetic Materials and Structures, Springer, Singapore (2015)

40. T.C. Lim, Phys. Status Solidi B 252, 1575-1579 (2015)

41. P. Sobieszczyk, M. Majka, D. Kuźma, T.C. Lim, and Piotr Zieliński, Phys. Status Solidi B 252, 1615-1619 (2015) 